\title{
Prospective Single-Center Study of SuperPulsed Thulium Fiber Laser in Retrograde Intrarenal Surgery: Initial Clinical Data
}

\author{
Mark Taratkin ${ }^{a} \quad$ Camilla Azilgareevab ${ }^{b}$ Dmitry Korolev ${ }^{a}$ Yazeed Barghouthy ${ }^{c}$ \\ Dmitry Tsarichenko ${ }^{a}$ Gagik Akopyan ${ }^{a}$ Denis Chinenov ${ }^{a}$ Stanislav Ali $^{a}$ \\ Vasiliy Kozlov ${ }^{d}$ Vasiliy Mikhailov ${ }^{a}$ Dmitry Enikeev ${ }^{a}$

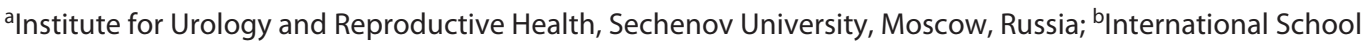 \\ "Medicine of the Future", Sechenov University, Moscow, Russia; 'GRC \#20 Lithiase Urinaire, Sorbonne University, \\ Hôpital Tenon, Paris, France; 'Department of Public Health and Healthcare Organization, Sechenov University, \\ Moscow, Russia
}

\section{Keywords}

Kidney · Retrograde intrarenal surgery · Thulium fiber laser · Urinary stones · Urolithiasis

\begin{abstract}
Introduction: The objective of this study was to present our clinical experience of using the thulium fiber laser in retrograde intrarenal surgery (RIRS). Methods: A prospective clinical study performed after the IRB approval (Sechenov University, Russia). Patients with stones $<30 \mathrm{~mm}$ were treated with SuperPulsed thulium fiber laser (SP TFL) (NTO IRE-Polus, Russia) through a 200- $\mu \mathrm{m}$-diameter fiber. Stone size, density, the duration of the operation, and laser on time (LOT) were measured. Based on the surgeon's feedback, retropulsion and intraoperative visibility were also assessed (Likert scale). Stone-free rates (SFRs) were assessed with a low-dose CT scan 90 days after the operation. Results: Between January 2018 and December 2019, 153 patients (mean age $54 \pm 2.8$ years) underwent RIRS with SP TFL (mean stone density $1,020 \pm 382 \mathrm{HU}$ ). Median stone volume was 279.6 (139.4615.8) $\mathrm{mm}^{3}$. Median LOT was 2.8 (IQR 1.6-6.6) min with median total energy for stone ablation $4.0($ IQR $2.1-7.17) \mathrm{kJ}$, median ablation speed was $1.7(1.0-2.8) \mathrm{mm}^{3} / \mathrm{s}$, median abla-
\end{abstract}

tion efficacy was $13.3(7.3-20.9) \mathrm{J} / \mathrm{mm}^{3}$, and energy consumption was $170.3(59.7-743.3) \mathrm{J} / \mathrm{s}$. Overall, the SFR (at 3 months) was $89 \%$. The overall complication rate was $8.4 \%$. Retropulsion was present in $23(15.1 \%)$ patients. Visibility was estimated as optimal in most patients, with poor visibility reported in only 13 (8.5\%) patients. Conclusion: The SP TFL is a safe and efficient tool in lithotripsy, irrespective of the stone type and density. Retropulsion is minimal and visibility is maintained with SP TFL. Nonetheless, further clinical studies are needed to ensure optimal comparison with conventional holmium:YAG lithotripsy.

(C) 2021 The Author(s).

Published by S. Karger AG, Basel

\section{Introduction}

Laser lithotripsy is an established first-line modality in treating renal calculi $[1,2]$. Over the last 2 decades, and thanks to major advances in endoscopic technology, flexible ureteroscopes have improved considerably and become widely available. Today, flexible ureteroscopy is the recommended treatment option for small $(<2 \mathrm{~cm})$ renal stones with high stone-free rates (SFRs) and low patient morbidity [3].
(C) 2021 The Author(s)

Published by S. Karger AG, Basel

This is an Open Access article licensed under the Creative Commons Attribution-NonCommercial-4.0 International License (CC BY-NC) (http://www.karger.com/Services/OpenAccessLicense), applicable to the online version of the article only. Usage and distribution for commercial purposes requires written permission.
Correspondence to:

Dmitry Enikeev, dvenikeev@gmail.com 
The holmium:YAG (Ho:YAG) laser is the standard laser source for lithotripsy today [4]. Introduced almost 30 years ago [5], this laser has benefited from years of subsequent technical development, and a great deal has been learnt from using it over the years. The Ho:YAG laser is capable of treating all urinary stone compositions, and in addition to its role in lithotripsy, it is used in the treatment of upper tract urothelial tumors.

In recent years, a new generation of lasers has evolved, thanks mainly to advances in laser fiber technology. Among these novel lasers, the main one is the SuperPulsed thulium fiber laser (SP TFL). This can operate within a large range of energy, frequency, and pulse duration settings [6-8]. Thulium fibers are made up of very thin silica fiber $(10-20 \mu \mathrm{m}$ core diameter and $50-200 \mu \mathrm{m}$ proximal fiber diameter). Miniaturization of the fiber is one of the main advantages of this laser for it allows better endoscope performance, through easier deflection, and enlarges the free space in the working channel [9]. Furthermore, the TFL produces smaller fragment size, with some studies showing 3-4 times more dust with TFL than with the Ho:YAG laser operating at similar power levels [10]. The aim of this study is to demonstrate, in a clinical setting, the efficacy and safety of SP TFL in retrograde intrarenal surgery (RIRS).

\section{Materials and Methods}

After having obtained the approval from the Institutional Review Board, a prospective clinical study was carried out (Sechenov University, Russia). A total of 153 patients with single renal calculi were treated with SP TFL (NTO IRE-Polus, Russia), using 200 or $400 \mu \mathrm{m}$ laser fibers. The procedures were performed by 4 surgeons experienced in laser lithotripsy, between January 2018 and December 2019. Patients included in the study had a single renal stone with a size up to $30 \mathrm{~mm}$, referred for RIRS. Exclusion criteria were anticoagulant or antiplatelet therapy, the need for secondary simultaneous surgical intervention (for benign prostatic hyperplasia, upper tract carcinoma, and urethral and ureteral stricture), and history of previous stone surgery or SWL.

Prior to inclusion into the study, all patients underwent contrast-enhanced computed tomography of the urinary tract, thus allowing the assessment of stone parameters, such as stone size and density (HU). All patients were pre-stented in 4-7 days before the procedure, prior to the subsequent use of a ureteral access sheath (10-12, Cook, USA), according to the accepted practice in our institution.

During the procedures, 2 flexible ureteroscopes were used: the 7.5 Fr. FLEX-X2 (Karl Storz GmbH Germany) and the single-use ureteroscope (LithoVue ${ }^{\mathrm{TM}}$; Boston Scientific, Marlborough, MA, USA). Intraoperative gravity irrigation was used in all patients (60-70 cm from the level of the patients' body). At the end of the procedure, a ureteral (double J) stent was placed up to 14 days
Table 1. Patient characteristics

\begin{tabular}{lc}
\hline Parameter & All patients $(n=153)$ \\
\hline Gender, male (\%)/female (\%) & $69(45) / 84(55)$ \\
Age, years & $54 \pm 2.8(18-80)$ \\
Kidney, right (\%)/left (\%) & $74(48) / 79(52)$ \\
Location, pelvis (\%)/calyx (\%) & $102(77) / 51(33)$ \\
Stone size, mm & $12.5 \pm 8.8(7-30)$ \\
Stone volume, mm $\mathrm{mm}^{3}$ & $279.6(139.4-615.8)$ \\
Density, $<1,000 \mathrm{HU}(\%) />1,000 \mathrm{HU}(\%)$ & $66(43) / 87(57)$ \\
Stone density, HU & $1,020 \pm 382(250-1,900)$ \\
\hline
\end{tabular}

Data presented as mean \pm SD (range) or median (IQR) where appropriate. SD, standard deviation.

postoperatively, according to the accepted practice in our institution. The bladder was drained with a Foley catheter for 1 day.

During the surgery, the operative time (from instrument insertion until removal) and laser on time (LOT, total time of laser ablation, automatically measured and demonstrated by the laser machine) were registered. To assess stone retropulsion and intraoperative visibility, surgeons were asked to rank them according to the 3-point Likert scale: 0 - excellent (absence of retropulsion and clear visibility), 1 - moderate (appropriate retropulsion and visibility), and 2 - poor (high retropulsion or low visibility, requiring to interrupt the procedure temporarily). The researcher (R.K.) accompanied the surgeons and aided in regimen selection, visibility, and retropulsion estimation. If any discrepancies in Likert scaling between the surgeon and R.K. were identified, the record of the surgery was reviewed by other surgeons taking part in this research. In addition, a consensus between the surgeons regarding the scaling was agreed upon to maximize the uniformity of the results.

Postoperative SFR was assessed with low-dose computed tomography 3 months after surgery. Patients without residual fragments, or with fragments smaller than $2 \mathrm{~mm}$ on CT at 3 months, were considered to be stone-free. The 3 size dimensions of stones were measured on $\mathrm{CT}$, allowing us to calculate the approximate stone volume according to the ellipsoid volume formula. Followup low-dose CT and ultrasonography were performed at 3 months to ensure that there was no stricture or hydronephrosis. Postoperative complications were classified according to the ClavienDindo classification [11].

The primary objectives of the study were to evaluate the safety of SP TFL lithotripsy, and its efficiency was measured through the following parameters: ablation speed $\left(\mathrm{mm}^{3} / \mathrm{s}\right)$, ablation efficacy $(\mathrm{J} /$ $\mathrm{mm}^{3}$ ), and the energy consumption (J/s). The secondary objectives were to evaluate the retropulsion, visibility, and complication rates.

\section{Statistical Analysis}

In terms of statistical analysis, we used SPSS Statistics 23.0 (IBM, Armonk, NY, USA). Mean and standard deviation values, median, and quartile distributions were calculated for variables. The Kolmogorov-Smirnov and Shapiro-Wilk tests were used for distribution assessment, Student $t$ test or Kruskal-Wallis and 
Table 2. Intraoperative data

\begin{tabular}{|c|c|c|c|c|}
\hline Stone density group & $\begin{array}{l}\text { All patients } \\
(n=153)\end{array}$ & $\begin{array}{l}\geq 1,000 \mathrm{HU} \\
(n=87)\end{array}$ & $\begin{array}{l}<1,000 \mathrm{HU} \\
(n=66)\end{array}$ & $\begin{array}{l}p \text { value } \\
(\geq 1,000 \mathrm{HU} \\
\text { vs. } \\
<1,000 \mathrm{HU})\end{array}$ \\
\hline Stone density, mean \pm SD (range), $\mathrm{HU}$ & $1,020 \pm 382(250-1,900)$ & $1,305.9 \pm 194.1(1,000-1,900)$ & $657.1 \pm 205.7(250-980)$ & $<0.001$ \\
\hline Stone size, mean $\pm \mathrm{SD}$ (range), $\mathrm{mm}$ & $12.5 \pm 8.8(7-30)$ & $10.5 \pm 4.9(9-30)$ & $11.7 \pm 6.1(7-30)$ & 0.171 \\
\hline Stone volume, median (IQR), $\mathrm{mm}^{3}$ & $279.6(139.4-615.8)$ & $263.9(146.6-460.8)$ & $351.1(131.9-754.0)$ & 0.057 \\
\hline Total energy, median (IQR), kJ & $4.0(2.1-7.2)$ & $3.9(2.3-6.8)$ & $4.0(2.0-7.2)$ & 0.371 \\
\hline Ablation speed, median (IQR), $\mathrm{mm}^{3} / \mathrm{s}$ & $1.7(1.0-2.8)$ & $1.3(0.9-2.5)$ & $2.1(1.2-3.5)$ & 0.508 \\
\hline Ablation efficacy, median (IQR), $\mathrm{J} / \mathrm{mm}^{3}$ & $13.3(7.3-20.9)$ & $16.2(8.6-22.8)$ & $11.5(6.8-16.8)$ & 0.054 \\
\hline Energy consumption, median (IQR), J/s & $170.3(59.7-743.3)$ & $160.1(64.8-593.5)$ & $189.0(58.1-775.2)$ & 0.303 \\
\hline
\end{tabular}

Data presented as mean \pm SD (range) or median (IQR) where appropriate. LOT, laser on time; SD, standard deviation.

Mann-Whitney $U$ tests were used where appropriate. We performed a correlation analysis (Spearman and Pearson correlation analysis) as well as a multivariable logistic regression analysis to determine any connections between laser and stone characteristics. A $p$ value of 0.05 was chosen as a statistically significant threshold.

\section{Results}

The study included 153 patients with a mean age of 54 years. The median LOT was $2.8(1.6-6.6) \mathrm{min}$, with a mean stone density of $1,020 \pm 382(150-1,900) \mathrm{HU}$, mean stone size was $12.5 \pm 8.8(7-30) \mathrm{mm}$, median stone volume was $279.6(139.4-615.8) \mathrm{mm}^{3}$ (Table 1). The median total energy for stone ablation was $4.0(2.1-7.17) \mathrm{kJ}$, the median ablation speed was $1.7(1.0-2.8) \mathrm{mm}^{3} / \mathrm{s}$, the median ablation efficacy was $13.3(7.3-20.9) \mathrm{J} / \mathrm{mm}^{3}$, and the energy consumption was 170.3 (59.7-743.3) J/s (Table 2). The mean postoperative stay was 3 days.

Two subgroups of patients were identified according to stone density. In the first subgroup, 66 patients (43.2\%), the stone density was $<1,000 \mathrm{HU}$, with a median LOT of 2.7 (1.5-6.8) min. The mean stone density was $657.1 \pm$ 205.7 (250-980) HU, the median stone volume was 351.1 (131.9-754.0) $\mathrm{mm}^{3}$, the median total energy for stone ablation was $4.0(2.0-7.2) \mathrm{kJ}$, the median ablation speed was $2.1(1.2-3.5) \mathrm{mm}^{3} / \mathrm{s}$, the median ablation efficacy was $11.5(6.8-16.8) \mathrm{J} / \mathrm{mm}^{3}$, and the energy consumption was 189.0 (58.1-775.2) J/s. In the second subgroup, 87 (56.8\%) patients with higher density stones $(\geq 1,000 \mathrm{HU})$ were identified. The median LOT was 2.9 (1.7-5.6) min. The mean stone density was $1,305.9 \pm 194.1(1,000-1,900)$ $\mathrm{HU}$, the median stone volume was 263.9 (139.4-463.4) $\mathrm{mm}^{3}$, the median total energy for stone ablation was 3.9 $(2.3-6.8) \mathrm{kJ}$, the median ablation speed was $1.3(0.9-2.5)$ $\mathrm{mm}^{3} / \mathrm{s}$, the median ablation efficacy was $16.2(8.6-22.8)$ $\mathrm{J} / \mathrm{mm}^{3}$, and the energy consumption was $160.1(64.8-$ 593.5) J/s (Table 2).

Retropulsion affected the continuation of the procedure (Likert score 2 ) in only $2(1.3 \%)$ patients, both of whom had renal pelvis stones. This resulted in prolongation of stone ablation time. Insignificant retropulsion was noted in 23 (15.1\%) patients (Likert score 1), whereas in the remaining $128(83.6 \%)$ cases, no retropulsion was reported (Likert score 0 ). Visibility was estimated to be optimal by surgeons in most cases, with only 13 (8.5\%) cases reporting poor/insufficient visibility (Likert score $1-2)$.

SFR at 3 months after procedure (residual fragments $<2 \mathrm{~mm}$ ) was $89 \%$ (136 of 153 patients). Seventeen patients (11\%) had fragments measuring between 2 and $4 \mathrm{~mm}$. The mean residual fragments' size was $2.5 \pm 0.8 \mathrm{~mm}$.

On the basis of a multivariable statistical analysis, the density (OR $=0.998,95 \%$ CI: 0.998-0.999, $p<0.001)$ and frequency $(\mathrm{OR}=1.005,95 \% \mathrm{CI}: 1.001-1.010, p<0,001)$ were closely correlated with poorer visibility. A multivariable analysis that adjusted for retropulsion showed a significant correlation with frequency $(\mathrm{OR}=0.987,95 \%$ CI: 0.977-0.997, $p=0.008$ ) (Table 3).

The Spearman and Pearson (used respectively) analyses showed that there was a significant correlation between LOT, stone volume $(r=0.733$ with $p<0.001)$, and total energy $(r=0.922$ and $p<0.001)$. It should be noted that we also identified a significant correlation between frequency and stone density $(r=0.245, p=0.001)$ and decreased visibility $(r=0.334, p<0.001)$ (Table 4$)$. 
Table 3. Uni- and multivariable analyses to measure the probability of poorer visibility and retropulsion

\begin{tabular}{|c|c|c|c|c|c|c|c|c|}
\hline & \multicolumn{4}{|c|}{ Univariable } & \multicolumn{4}{|c|}{ Multivariable } \\
\hline & $p$ value & OR & \multicolumn{2}{|c|}{$95 \% \mathrm{CI}$} & $p$ value & OR & \multicolumn{2}{|c|}{$95 \% \mathrm{CI}$} \\
\hline \multicolumn{9}{|l|}{ Visibility } \\
\hline Density & $<0.001$ & 0.999 & 0.998 & 0.999 & $<0.001$ & 0.998 & 0.998 & 0.999 \\
\hline Stone volume & 0.021 & 1.000 & 0.999 & 1.000 & 0.401 & 1.000 & 1.000 & 1.001 \\
\hline Frequency & $<0.001$ & 0.994 & 0.991 & 0.997 & 0.019 & 1.005 & 1.001 & 1.010 \\
\hline Ablation speed & $<0.001$ & 0.587 & 0.485 & 0.710 & 0.117 & 0.792 & 0.592 & 1.060 \\
\hline \multicolumn{9}{|l|}{ Retropulsion } \\
\hline Density & $<0.001$ & 0.998 & 0.998 & 0.999 & 0.393 & 1.000 & 0.999 & 1.000 \\
\hline Stone volume & 0.006 & 0.999 & 0.999 & 1.000 & 0.762 & 1.000 & 0.999 & 1.001 \\
\hline Frequency & $<0.001$ & 0.969 & 0.957 & 0.981 & 0.008 & 0.987 & 0.977 & 0.997 \\
\hline Ablation speed & $<0.001$ & 0.363 & 0.265 & 0.496 & 0.059 & 0.622 & 0.380 & 1.018 \\
\hline
\end{tabular}

Values in bold indicate significant correlation.

Table 4. Pearson and Spearman correlations between the stones' characteristics and laser settings

\begin{tabular}{|c|c|c|c|c|c|c|c|}
\hline Parameter & Stone density & Stone volume & LOT & Frequency & $\begin{array}{l}\text { Ablation speed } \\
\left(\mathrm{mm}^{3} / \mathrm{s}\right)\end{array}$ & $\begin{array}{l}\text { Ablation efficacy } \\
\left(\mathrm{J} / \mathrm{mm}^{3}\right)\end{array}$ & $\begin{array}{l}\text { Energy } \\
\text { consumption }(\mathrm{J} / \mathrm{s})\end{array}$ \\
\hline Stone density & - & $-0.158(0.054)$ & $0.004(0.964)$ & $0.245(0.001)^{*}$ & $-0.270(0.001)^{*}$ & $0.210(0.010)^{*}$ & $0.019(0.818)$ \\
\hline LOT & $0.004(0.964)$ & $0.733(<0.001)^{*}$ & - & $0.007(0.934)$ & $-0.036(0.660)$ & $0.076(0.351)$ & $0.981(<0.001)^{*}$ \\
\hline Total energy & $0.013(0.877)$ & $-0.648(<0.001)^{*}$ & $0.922(<0.001)^{*}$ & $0.098(0.233)$ & $-0.008(0.921)$ & $0.140(0.087)$ & $0.976(<0.001)^{*}$ \\
\hline Visibility & $-0.34(0.686)$ & $0.167(0.041)^{*}$ & $0.145(0.076)$ & $0.334(<0.001)^{*}$ & $0.050(0.545)$ & $-0.075(0.361)$ & $0.155(0.057)$ \\
\hline
\end{tabular}

Presented as Pearson or Spearman (used where appropriate) coefficient ( $p$ value). LOT, laser on time. * Significant correlation.

The postoperative complication rate was low, with Clavien I-II complications present in 13 (8.4\%) patients (Table 5). No complications specific to the use of TFL were identified. No signs suggesting strictures or stenoses were identified on CT imaging at 3 months after surgery.

\section{Discussion}

In this clinical trial, we were able to demonstrate that SP TFL is a safe and efficient tool for lithotripsy. The higher frequency setting allowed for fast stone dusting and did not result in any increase in complications. SP TFL stone fragmentation was characterized by short LOT, good visibility, and minimal retropulsion, irrespective of the stone type, thus indicating that thulium fiber is highly efficient in a large variety of stone densities.
Table 5. Complications

\begin{tabular}{lc}
\hline Complication & Total $(n=153)$ \\
\hline Grade I & \\
$\quad$ Fever, $n(\%)$ & $6(3.9)$ \\
$\quad$ Transient creatinine elevation, $n(\%)$ & $4(2.6)$ \\
Grade II & \\
$\quad$ UTI, $n(\%)$ & $3(1.9)$ \\
Overall & $13(8.4)$ \\
\hline
\end{tabular}

In this study, the achieved median LOT of 2.7 (IQR 1.6-6.6) min indicates that the TFL can be as effective in upper urinary tract lithotripsy as Ho:YAG. In a previous study, Ibrahim et al. [6] showed that in an in vitro study on 5-mm artificial stones, when using Ho:YAG laser with Moses technology, a mean LOT of 5.3 min was achieved. Ventimiglia et al. [7] compared SP TFL with Ho:YAG and 
the effects of pulse shape in controlled in vitro studies. This study found that SP TFL resulted in lower retropulsion and speed of stone movement, while the short-pulse mode of the Ho:YAG laser gave rise to the greatest stone displacement $(p<0.001)$ and stone movement $(p<0.001)$. Moreover, the ablation efficiency was higher for the SP TFL than for the Ho:YAG laser.

TFL, as mentioned previously, is a thin silica fiber, with an emission from the uniform laser beam at the output, with a wavelength of $1,940 \mathrm{~nm}$, in comparison to the $2,120 \mathrm{~nm}$ wavelength of Ho:YAG laser [8]. Given that the absorption peak for water is around $1,940 \mathrm{~nm}$, it seems that one of the main advantages of SP TFL is that its wavelength lies very close to this peak. Hence, based on the wavelength consideration alone, it is expected that the TFL can be up to 4-5 times more efficient in stone ablation than the Ho:YAG laser for similar settings $[9,10,12$, 13]. Previous preclinical studies demonstrated that SP TFL allows for up to 4 times better ablation efficacy, with significant reduction in retropulsion, which may facilitate its efficacy during RIRS $[10,12]$. It is worth mentioning that the high frequency utilized in certain TFL settings raised concerns regarding the potential rise of temperature in the irrigation solution. However, Andreeva et al. [10] showed that when comparing TFL with Ho:YAG lasers, there was no difference in the change of temperature between the 2 lasers [12]. The study findings were supported by Taratkin et al. [14], who showed a comparable temperature increase in SP TFL and Ho:YAG. Thus, greater energy absorption did not result in a higher temperature rise during the procedure.

In the current trial, we observed a reduced retropulsion of SP TFL. Significant retropulsion was observed in 1.3\% of patients. In all of the other cases, the surgeons reported minimal to no retropulsion. It should be mentioned, however, that while reduced retropulsion may aid the surgeon, it does not necessarily increase stone ablation efficiency or shorten the duration of the operation. Similar reduction in retropulsion was also described previously when using Ho:YAG laser and Moses technology [15]. SP TFL also allowed for better visibility in the vast majority of cases (poor visibility was reported in only 13 of 153 cases).

At 3 months, we found that $89 \%$ of patients were stonefree, with only 5 patients (3.3\%) showing residuals larger than $3 \mathrm{~mm}$. These results are comparable to those reported in the SFR literature when using CT as an imaging modality for control [15-20]. Furthermore, the postoperative SFR was assessed using low-dose computed tomography 3 months after the surgery, which was considered to be the optimal method in comparison to US or KUB [21].
Another important issue to be addressed is that of renal pelvis damage with SP TFL. To assess the safety of the device, all surgeries were reviewed by an experienced endourologist to be sure that there was no damage due to increased temperature or direct laser ablation. Mild mucosal injuries occurred several times during the stone ablation. However, no cases of bleeding or extensive damage were recorded in our study. Indeed, the safety of TFL in soft tissue surgery has previously been well studied $[22,23]$. This could point to an additional potential advantage of SP TFL, which is that TFL affects only superficial mucosal layers without any damages to deeper layers due to the greater absorption of laser energy in water. In our study, the overall complication rate was low, as demonstrated in Table 2, and no serious complications (Clavien-Dindo 3-4) were identified.

This is one of the first clinical studies to be published and one of the largest cohorts so far to be treated with TFL, demonstrating its efficiency and safety. The fact that 4 different surgeons took part in this study reduced the likelihood that the results were biased. Furthermore, it allowed for a more diverse perspective on laser performance and a more reliable mean retropulsion and visibility scores. The fact that a researcher participated in all the surgeries and discussed the final score of each scale with surgeons, allowed us to reduce inter-operator variability and to present data with the minimal bias possible.

The main limitations of our study were the absence of a control group for comparison of our results and the subjective assessment of stone retropulsion by the surgeons using visual reporting for retropulsion. However, measuring retropulsion accurately, which has been done in laboratory conditions, using a high-speed camera that recorded the stone retropulsion along a ruler [24] is limited in an in vivo setup without sophisticated graphical programs to measure the fine retropulsion movements. Also, it should be noted that stone composition was not presented in the current trial. However, stone density was assessed in all patients, providing reliable data on laser efficacy in different stone types. Another important point that should be mentioned is that both reusable and singleuse scopes were used in the study. We believe that it did not affect the laser working parameters and/or the results of the study. Furthermore, it has been backed up with data from other clinical trials [25], suggesting similar results of surgery with single-use and reusable ureteroscopes. Another limitation was the heterogeneous population with their different stone densities and sizes and different surgical training capacities of the 4 different surgeons. Pre-stenting, although not a commonly recommended practice worldwide, is carried out in our institute. The 
lack of a control group or similar clinical studies in other centers with a different practice prevents us from ascertaining whether this might have influenced our results. Nevertheless, as was mentioned previously, our primary objective was to assess the thulium fiber efficiency and safety. Despite the heterogeneous nature of the group, we are confident that we achieved this goal.

\section{Conclusion}

The SP TFL is a safe and efficient tool in lithotripsy, irrespective of the stone type and density. Retropulsion is minimal and visibility is maintained with SP TFL. Nonetheless, further clinical studies are needed for optimal comparison with conventional Ho:YAG lithotripsy.

\section{Statement of Ethics}

The study was approved by the Sechenov University (Moscow, Russia) Institutional Review Board. Informed consent was obtained from all individual participants included in the study.

\section{Conflict of Interest Statement}

The authors have stated explicitly that there are no conflicts of interest in connection with this article.

\section{Funding Sources}

This research did not receive any specific grant from funding agencies in the public, commercial, or not-for-profit sectors.

\section{Author Contributions}

M.T.: data analysis and manuscript writing. C.A.: data analysis and manuscript writing. D.K.: data analysis and manuscript editing. Y.B.: manuscript editing. D.T.: data collection and manuscript editing. G.A.: protocol development and data collection. D.C.: protocol development and data collection. S.A.: data collection and data analysis. V.K.: data analysis and manuscript writing. V.M.: protocol development and manuscript editing. D.E.: data analysis, manuscript editing, protocol development, and management.

\section{Availability of Data and Material}

On demand.

\section{References}

1 Raheem OA, Khandwala YS, Sur RL, Ghani KR, Denstedt JD. Burden of urolithiasis: trends in prevalence, treatments, and costs. Eur Urol Focus. 2017 Feb;3(1):18-26.

2 Scales CD, Smith AC, Hanley JM, Saigal CS. Prevalence of kidney stones in the United States. Eur Urol. 2012;62(1):160-5.

3 Giusti G, Proietti S, Villa L, Cloutier J, Rosso M, Gadda GM, et al. Current standard technique for modern flexible ureteroscopy: tips and tricks. Eur Urol. 2016 Jul;70(1):188-94.

4 Bell JR, James P, Rane A, Nakada SY. International holmium laser lithotripsy settings: an international survey of endourologists. J Am Coll Surg. 2016.

5 Doizi S, Keller EX, De Coninck V, Traxer O. Dusting technique for lithotripsy: what does it mean? Nature reviews urology. Nature Publishing Group; 2018. Vol. 15; p. 653-4.

6 Ibrahim A, Badaan S, Elhilali MM, Andonian S. Moses technology in a stone simulator. Can Urol Assoc J. 2018;12(4):127.

7 Ventimiglia E, Doizi S, Kovalenko A, Andreeva V, Traxer O. Effect of temporal pulse shape on urinary stone phantom retropulsion rate and ablation efficiency using holmium: YAG and super-pulse thulium fibre lasers. BJU Int. 2020.
8 Chan KF, Pfefer TJ, Teichman JMH, Welch AJ. A perspective on laser lithotripsy: The fragmentation processes. J Endourol. 2001; 15:257-73.

9 Taratkin M, Laukhtina E, Singla N, Tarasov A, Alekseeva T, Enikeev M, et al. How lasers ablate stones: in vitro study of laser lithotripsy (Ho:YAG and Tm-Fiber Lasers) in different environments. J Endourol. 2020 Jan 29.https: //www.liebertpub.com/doi/10.1089/ end.2019.0441.

10 Andreeva V, Vinarov A, Yaroslavsky I, Kovalenko A, Vybornov A, Rapoport L, et al. Preclinical comparison of superpulse thulium fiber laser and a holmium:YAG laser for lithotripsy. World J Urol. 2020 Feb 1;38(2): 497-503.

11 Dindo D, Demartines N, Clavien PA. Classification of surgical complications: a new proposal with evaluation in a cohort of $6,336 \mathrm{pa}$ tients and results of a survey. Ann Surg. 2004 Aug;240(2):205-13.

12 Pasqui F, Dubosq F, Tchala K, Tligui M, Gattegno B, Thibault $\mathrm{P}$, et al. Impact on active scope deflection and irrigation flow of all endoscopic working tools during flexible ureteroscopy. Eur Urol. 2004;45(1):58.
13 Hardy LA, Vinnichenko V, Fried NM. High power holmium:YAG versus thulium fiber laser treatment of kidney stones in dusting mode: ablation rate and fragment size studies. Lasers Surg Med. 2019;51(6):522.

14 Taratkin M, Laukhtina E, Singla N, Kozlov V, Abdusalamov A, Ali S, et al. Temperature changes during laser lithotripsy with Ho:YAG laser and novel Tm-fiber laser: a comparative in-vitro study. World J Urol. 2020 Feb 20. http: //link.springer.com/10.1007/s00345020-03122-1.

15 Danilovic A, Torricelli FCM, Marchini GS Batagello C, Vicentini FC, Traxer O, et al. Prospective evaluation of bilateral retrograde intrarenal surgery: is it really safe? J Endourol. 2020 Sep 9;35(1):14.https://www.liebertpub. com/doi/10.1089/end.2020.0611.

16 Danilovic A, Cavalanti A, Rocha BA, Traxer O, Torricelli FCM, Marchini GS, et al. Assessment of residual stone fragments after retrograde intrarenal surgery. J Endourol. 2018 Dec 1;32(12):1108-13.

17 Bozzini G, Verze P, Arcaniolo D, Dal Piaz O, Buffi NM, Guazzoni G, et al. A prospective randomized comparison among SWL, PCNL and RIRS for lower calyceal stones less than $2 \mathrm{~cm}$ : a multicenter experience. World J Urol. 2017 Dec 5;35(12):1967-75. Available from: http://link. springer.com/10.1007/s00345-017-2084-7. 
18 Bhojani N, Paonessa JE, El Tayeb MM, Williams JC, Hameed TA, Lingeman JE. Sensitivity of noncontrast computed tomography for small renal calculi with endoscopy as the gold standard. Urology. 2018 Jul 1;117:36-40.

19 El-Nahas AR, Almousawi S, Alqattan Y, Alqadri IM, Al-Shaiji TF, Al-Terki A. Dusting versus fragmentation for renal stones during flexible ureteroscopy. Arab J Urol. 2019 Apr 3;17(2):138-42.

20 Lee YJ, Bak DJ, Chung JW, Lee JN, Kim HT, Yoo $\mathrm{ES}$, et al. Is it necessary to actively remove stone fragments during retrograde intrarenal surgery? Investig Clin Urol. 2016 Jul 1;57(4):274.
21 Pearle MS, Lingeman JE, Leveillee R, Kuo R, Preminger GM, Nadler RB, et al. Prospective, randomized trial comparing shock wave lithotripsy and ureteroscopy for lower pole caliceal calculi $1 \mathrm{~cm}$ or less. J Urol. 2005;173(6): 2005-9.

22 Enikeev D, Okhunov Z, Rapoport L, Taratkin M, Enikeev M, Snurnitsyna O, et al. Novel thulium fiber laser for enucleation of prostate: a retrospective comparison with open simple prostatectomy. J Endourol. 2019;33(1):16.
23 Enikeev D, Shariat SF, Taratkin M, Glybochko $\mathrm{P}$. The changing role of lasers in urologic surgery. Curr Opin Urol. 2020 Jan 1;30(1): 24-9.

24 Traxer O, De Coninck V, Keller E, Kovalenko A, Andreeva V, Doizi S. Comparing short, long, and Moses regimes of Ho:YAG laser versus Super PulseTM fiber laser in vitro: ablation speed and retropulsion effect. Eur Urol Suppl. 2019.

25 Davis NF, Quinlan MR, Browne C, Bhatt NR, Manecksha RP, D’Arcy FT, et al. Singleuse flexible ureteropyeloscopy: a systematic review. World J Urol. 2018 Apr;36(4):52936. 